\title{
Panarchie dans le Sahel
}

\author{
L'initiative de la Grande Muraille Verte au prisme \\ des relations d'influence entre acteurs
}

\author{
Axel Ducourneau', Abdou Ka², \\ Mehdi Saqallli ${ }^{3}$
}

\begin{abstract}
[Résumé] Cet article porte sur l'initiative de la «Grande muraille verte», projet de reforestation et de développement intégré de la bande sahélienne et sur la mise en place d'un observatoire dédié, au Sénégal. Sur base d'une observation participante, les auteurs analysent le passage de la rhétorique environnementale à l'action opérationnelle sur le terrain à travers le prisme des mécanismes d'action collective mis en œuvre. On s'aperçoit que, sur le terrain, la capacité de négociations des acteurs est dépendante de plusieurs types de contingences : la configuration géographique du site du projet, l'historique du site et des projets qui s'y sont succédé au cours des 50 dernières années, la personnalité des acteurs et les intérêts propres des groupes en présence. Le concept de gouvernance apparaît alors comme un paradigme qui recouvre l'ensemble des négociations interpersonnelles enclenchées pour aboutir à un objectif concret : la mise en place de parcelles reforestées. Finalement, les auteurs montrent que le thème global de la désertification permet la mobilisation de fonds financiers internationaux et la rhétorique de la gouvernance, dont la définition est laissée volontairement à l'interprétation de chacun des acteurs, permet quant à elle les ajustements d'objectifs plus ou moins partagés.
\end{abstract}

Mots-clés : Analyse de réseaux sociaux/Aide à la décision Cartographie participative Gouvernance multimodale Applications des politiques publiques

[Abstract] This article focuses on the Great Green Wall Initiative, a reforestation project in Sahel and the implementation of its monitoring in Senegal. Based on a "participatory" observation as an actor involved in this project, this project appears as emblematic of the projects referring to many legitimacies (environmentalism, transnational cooperation, national legitimization, local development, scientific rigor) while each of them is questioned. Beyond the associated rhetoric, conducting this project means practically following a gentle avoidance of every possible point of friction: On the reforestation site, the local management mode goes through a non-formalized entanglement of the state and local communities. The theme of desertification first allows the mobilization of international funds. The rhetoric of governance sees its definition intentionally left to the interpretation of each player where each stakeholder find a profit to maintain open dialogue. Scientists, regardless of nationality, are then the de facto interface between these spheres. Even more, the management of scientific

\footnotetext{
${ }^{1}$ Université de la Nouvelle-Calédonie, UMR 7268 ADES AMU-CNRS-EFS.

2 Université Cheikh Anta Diop de Dakar, Umi 3189 ESS « Environnement, Santé, Sociétés ».

${ }^{3}$ CNRS, UMR 5602 GEODE « Géographie de l'Environnement ».
} 
disciplines, for the same financial and political reasons, follows the same strategy of conflict avoidance and tacit control.

Keywords: social network analysis decision-making / participative mapping / multimodal governance / public policy practices

\section{Gouvernance et problématique des décisions multi-échelles}

La désertification du Sahel est un enjeu scientifique et politique international depuis le début des années 1980. Il ne cesse de prendre de l'importance dans le contexte actuel du changement climatique, et il devient aujourd'hui un véritable enjeu de diplomatie scientifique, certes comme une volonté de lutter contre la dégradation des conditions socio-économiques des populations de la zone, mais aussi comme une résurgence du panafricanisme dans un environnement géopolitique sahélien complexe. L'enjeu environnemental de cette zone est avant tout la survie des populations qui s'y trouvent.

L'objectif de cet article est d'explorer, à partir de l'exemple de la mise en œuvre d'un observatoire scientifique pluridisciplinaire en Afrique de l'Ouest, le mode de gouvernance au sein du projet panafricain de la Grande Muraille Verte pour le Sahara et le Sahel (GMV). Il s'agit d'évaluer l'importance des relations interpersonnelles dans les rouages d'actions collectives imaginées de manière transnationale et appliquées au niveau régional et local.

L'hypothèse de travail est que les modalités de l'action collective liées à la rhétorique institutionnelle du projet transnational GMV («lutter contre la désertification») varient selon l'échelle observée du projet et que l'enchevêtrement des modes de gouvernance a une répercussion sur la manière dont les actions associées sont conçues puis pratiquées.

À ce titre, le concept de gouvernance est central. Nous reprendrons une définition opérationnelle, communément acceptée par les divers acteurs des projets de développement, celle du Programme des Nations Unies pour le Développement : «la gouvernance correspond à l'exercice de l'autorité politique, économique et administrative dans la gestion des affaires du pays à tous les niveaux - global, central, régional et local. Elle comprend les mécanismes complexes, les processus et les institutions au travers desquelles les citoyens, individuellement ou en association, et les personnes morales gèrent les intérêts, exercent leurs droits et obligations et négocient leurs désaccords. Sa responsabilité incombe non seulement à l'État, mais également au secteur privé et à la société civile». Créer des configurations de gouvernance environnementales qui permettent l'intégration des acteurs est un enjeu de politique publique majeur à l'échelle internationale. C'est aussi un objectif d'une profonde complexité sociale et opérationnelle, car il s'agit en fait de créer de l'action collective. Les facteurs économiques, écologiques et sociaux interfèrent dans les pratiques décisionnelles et, d'un point de vue scientifique, le transfert des connaissances à la société est dépendant de la capacité des institutions de recherche et des individus qui les composent à créer du lien social. 
Le projet de la Grande Muraille Verte pour le Sahara et le Sahel (GMV) est avant tout un processus politique à visée panafricaine concrétisé par l'objectif affiché de revégétaliser la bande sahélienne en proie à des cycles réguliers de sécheresses dont la plus emblématique remonte aux années 1980. L'initiative s'inscrit également dans les recommandations du Millennium Ecosystem Assessment visant à promouvoir des politiques intégrées de développement local en permettant de capter des financements internationaux. Après une mise en place complexe dans le climat géopolitique du Sahel, c'est finalement par une redistribution fléchée des fonds du Fond pour l'Environnement Mondial (FEM) dans les 11 pays concernés que le projet a pu voir le jour à partir de 2009. Le Sénégal en a d'emblée porté le leadership, car son président d'alors, Abdoulaye Wade, était également président du volet environnement du Nouveau Partenariat pour le Développement de l'Afrique (NEPAD), programme de l'Union africaine en charge des questions de développement du continent. En 2016, le G5 Sahel considère ce projet comme un des moyens d'émancipation des populations du Sahel contre l'influence des extrémismes, au premier lieu desquels, le terrorisme islamiste.

Cette complexité organisationnelle à laquelle les chercheurs participent eux-mêmes, a motivé nombre de travaux de recherche sur des configurations où acteurs hétéroclites interagissent de manière affichée ou cachée, formelle ou informelle, plus ou moins conflictuelle, mais sans possibilité d'éviter des rapports de pouvoir et de force (Weber, 1971). Ces travaux vont de l'analyse socio-anthropologique monographique d'actions de gouvernance (Lemieux, 1989), qu'elle soit environnementale ou de «développement» (Olivier de Sardan et al., 1995 ; Travaux du LASDEL, 1999 à aujourd'hui ; Bierschenk et al., 2000) à l'analyse en terme de théorie des jeux (Ostrom, 1990).

\section{Concepts, matériau d'étude et méthodologie}

Le contenu de cet article est fondé sur l'observation directe et sur des entretiens liés à nos travaux quotidiens respectifs, comme coordinateur du projet scientifique, scientifique associé sur un programme et étudiant au sein de l'observatoire OHM (Observatoire Hommes-Milieux) sur la période 2009 à 2013. Les données et figures présentées sont d'une part des formalisations géographiques via un Système d'Information Géographique (SIG : Figure 1) et d'autre part les résultats d'un travail d'analyse du réseau d'acteurs par un outil de formalisation de ce réseau qui ne sera représenté dans cet article que sous forme schématique (Broadbent, 2014 ; Figure 2)

Nous abordons la gouvernance à travers le prisme de l'action collective. La gouvernance du projet GMV est avant tout la construction sociale de partenariats multiformes et, dans notre cas, multiculturels. Elle inclut à la fois la construction d'un système de représentation sociale ayant pour but de maintenir l'image du projet dans la rhétorique institutionnelle et une pratique empirique fondée sur des relations d'acteurs individuels, issus de groupes variés (scientifiques, bailleurs, politiques, organisations non gouvernementales). Notre posture est celle de praticiens et de «participants 
observateurs » (Mosse, 2005), au sein d'un projet transnational. Cette posture implique inévitablement une remise en question professionnelle sur les implications d'une recherche-action qui se fait en contexte de collaboration multilatérale. Par ailleurs, malgré sa portée opérationnelle, cette posture réflexive n'a pas pour objectif de juger le fonctionnement d'un dispositif auquel nous avons participé, mais simplement d'en démêler les ressorts.

\section{Contexte scientifique de la Grande Muraille Verte au Sénégal}

La Grande Muraille verte pour le Sahara et le Sahel est un projet implanté dans une réserve sylvo-pastorale dans une région sahélienne, le Ferlo, parcourue par des éleveurs peuls. Jusqu'à la colonisation française, aucune implantation permanente n'existait. La mise en place de forages par le pouvoir colonial français en 1950 a permis un maillage de points d'eau modifiant profondément le régime des migrations semi-nomades. L'événement fondateur des modifications socioéconomiques locales est initialement la construction de forages dans une perspective conversationniste suivi par la succession de projets internationaux.

L'installation d'un observatoire international dans cette région du Sahel a une dimension politique et symbolique forte. Au-delà de l'image médiatisée et scientifiquement erronée d'une «muraille » contre la désertification, la question de la coordination des États du Sahel et des structures de recherche s'est posée rapidement. Au Sénégal, la zone a été définie par l'agence nationale de la GMV, créée en 2010, en charge de la mise en œuvre du projet. Cette zone, gérée par le service des Eaux et des Forêts du Ministère de l'Environnement, est connue pour l'importance des projets internationaux de développement (GIZ4, Programme Alimentaire Mondial, Food and Agriculture Organisation) et de suivis scientifiques qui s'y sont succédé. Le Centre International de Recherche en Agronomie pour le Développement (CIRAD) et l'Institut de Recherche pour le Développement (IRD), organismes de recherche français «à destination des pays du Sud », y étaient déjà implantés depuis plus d'une dizaine d'années. Pour des raisons d'opportunités de collaborations et de stratégies scientifiques à l'international, seul le CNRS, paradoxalement moins orienté "pays du Sud», s'est explicitement positionné dès 2009 sur le terrain, via la création d'un observatoire dédié au suivi pluridisciplinaire de la GMV.

Au fil des années, parallèlement à l'intérêt croissant des bailleurs (Banque Mondiale, FEM) et certains États comme la Turquie, les acteurs scientifiques français s'y sont repositionnés. Les collaborations au sein de l'observatoire et entre organismes français sont axées sur le co-financement de projets individuels d'étudiants ou de chercheurs essayant d'optimiser la dimension temporelle attendue d'un observatoire, à savoir la constitution d'une base de données diachronique et pérenne des résultats de recherche

${ }^{4}$ Deutsche Gesellschaft für Internationale Zusammenarbeit, ex-GTZ. 
obtenus. La stratégie a été de privilégier, d'abord par des relations informelles, puis par des accords contractuels de partenariats bilatéraux, les liens avec les agences de l'État sénégalais concernées, en premier lieu l'ANGMV et le centre de suivi écologique (CSE). Ce lien fort OHM-ANGMV-UCAD 5 est la vitrine nationale de l'action scientifique de l'OHM qu'il convenait de maintenir dans le Ferlo. Il permet de développer des projets, notamment en agroforesterie qui n'auraient aucune chance d'exister sans le soutien institutionnel et logistique de l'État sénégalais (Binswanger, 1986; Kristjanson et al., 2002 ; Poussin et al., 2006; Nederlof \& Dangbégnon, 2007; Parrott \& Meyer, 2012).

Dans la pratique, c'est bien le lien historique et personnel entre d'une part certains chercheurs français, porteurs du projet et affiliés à l'IRD et au CNRS et d'autre part certains enseignants-chercheurs de l'UCAD, qui constitue la colonne vertébrale du projet, chacun jouant le rôle de dirigeant, de porteur, de relais et de mobilisateur vis-à-vis des institutions publiques de son pays respectif.

Ainsi, l'implantation scientifique a une historicité forte à la fois dans le Ferlo et à Dakar, où se concentrent toutes les institutions sénégalaises et françaises. Cela a pour conséquences de créer un éventail d'opportunités d'actions, défini fortement par les relations entre acteurs déconnectées du contexte sahélien quotidien. La multiplicité des partenaires montre le poids des institutions dans la mise en place d'un projet qui se veut participatif et à l'écoute des populations, mais dont la conception est fondamentalement «top-down» (Blondiaux, 2004). Par ailleurs, suivant Lavigne-Delville (2001; 2011), il était important de comprendre ces jeux d'acteurs institutionnels et locaux dans leurs contextes historiques et géographiques, lesquels permettent un éclairage sur l'état des relations sociales de la zone étudiée.

\section{Historique des projets antérieurs}

En 1925, lors de la Conférence Economique du Sénégal, le problème de l'accès à l'eau est soulevé. L'Administration coloniale française s'inquiète des dangers d'une économie basée exclusivement sur la culture de l'arachide. Parallèlement, vers 1935 arrivent du Fuuta vers le Ferlo les premières familles Bissinaabe qui aujourd'hui constituent le clan majoritaire de la région de Widou Thiengoly. En juillet 1955, les premières implantations permanentes débutent autour du forage nouvellement construit de Widou Thiengoly. À partir de 1970, marquée par une grande sécheresse, s’installe un projet sénégaloallemand de reboisement de la zone nord qui fut le plus grand employeur de la région pendant près de 25 ans. Ces emplois créés expliquent en partie la ruée que connût le forage de Widou Thiengoly et sa transformation en village, accompagné d'une modification profonde des parcours migratoires saisonniers. Cette période sera, pour la population de Widou Thiengoly, le point de comparaison économique pour tous les projets qui suivront. C'est à partir de 2005, après une période de transition du projet

${ }^{5}$ Université Cheikh Anta Diop : basée à Dakar, principale université du pays. 
allemand qu’a été entrepris le projet GMV, reprenant partiellement la logistique et les locaux des anciennes initiatives.

Cette succession historique explique en partie le ressenti mitigé des populations environnantes voyant en la GMV un projet moins pourvoyeur en emploi et avantages directs (carburant gratuit par exemple). À l'échelle internationale, cette diminution apparente des moyens est plus simplement représentative de la dynamique économique fondée sur la réalisation d'objectifs des institutions du développement et de l'aide internationale dont la doctrine a évolué vers l'appui aux actions communautaires. Cependant, au niveau local, la stricte comparaison des bénéfices directs donne à la GMV un caractère mal proportionné aux besoins, voire inutile qui, même s'il est subjectif, rend toute nouvelle action de développement moins évidente à légitimer.

\section{Spatialisation des relations d'influences locales}

Dans le cadre de l'initiative GMV, les activités principales de lutte contre la désertification ont consisté à mettre en défens de parcelles associées à des campagnes de reforestation. Celles-ci demandent une logistique liée à la production de jeunes plants en pépinières qu'il faut arroser pendant 3 mois avant de les transférer à la saison des pluies sur les parcelles prédéterminées. Les parcelles doivent être à portée de véhicule et à distance raisonnable (entre 7 et $15 \mathrm{~km}$ ) des pépinières constituées dans les villages (figure 1). Il faut alors négocier les tracés des parcelles avec les propriétaires de campements en fonction des routes et des territoires traditionnellement utilisés pour le bétail afin que la surface d'implantation des parcelles, clôturées, sur 600 à 2000 hectares, ne rallonge pas le chemin des familles vers le forage. La stratégie de mise en œuvre place le tracé de la GMV le long d'un pare-feu entre les 2 principaux villages de la communauté rurale (figure 1).

Les agents locaux de l'État ont un rôle majeur dans ces négociations et une fois sur le terrain, à $500 \mathrm{~km}$ de la capitale, les liens hiérarchiques institutionnels auxquels sont soumis ces agents paramilitaires de l'ANGMV perdent de leur légitimité au profit d'une densification des liens locaux avec la communauté rurale (figure 2). L'usage des arrangements interpersonnels lors de la mise en œuvre des opérations sur le terrain (pour la définition des lieux d'implantation des parcelles par exemple) procède d'un transfert tacite de responsabilité vers les exécutants locaux qui se doivent de réaliser des plantations visibles par les bailleurs tout en évitant les conflits locaux. Que les réalisations répondent à des nécessités écologiques à long terme devient secondaire face à la paix sociale immédiate dans la communauté rurale. Dès lors, l'influence personnelle des acteurs devient un ressort puissant dans la configuration locale du projet : celle-ci étant ainsi établie sur des bases sociales et non environnementales, elle peut contraindre par ricochet les choix techniques et scientifiques de suivi des parcelles. Ainsi, c'est en partie les conflits locaux entre les clans peuls Bissinaabe et Bacarnaabe et leurs liens avec les agents de l'État, qui expliquent l'implantation géographique du projet GMV, un clan 
ayant été privilégié dans l'attribution des parcelles. Les habitants du secteur S7 (Bissinaabe) n'ont pas obtenu de parcelles alors que ceux du secteur S1 (Bacarnaabe) ont obtenu un grand nombre de parcelles dans la première phase du projet (figure 1).

Enfin, l'éclatement géographique des institutions participantes sur la bande sahélienne (7000 km de Dakar à Djibouti) et la délocalisation des centres de décisions favorisent également une certaine érosion de la motivation des acteurs et facilitent ce transfert de responsabilité dans le choix des lieux d'implantations des actions du projet.

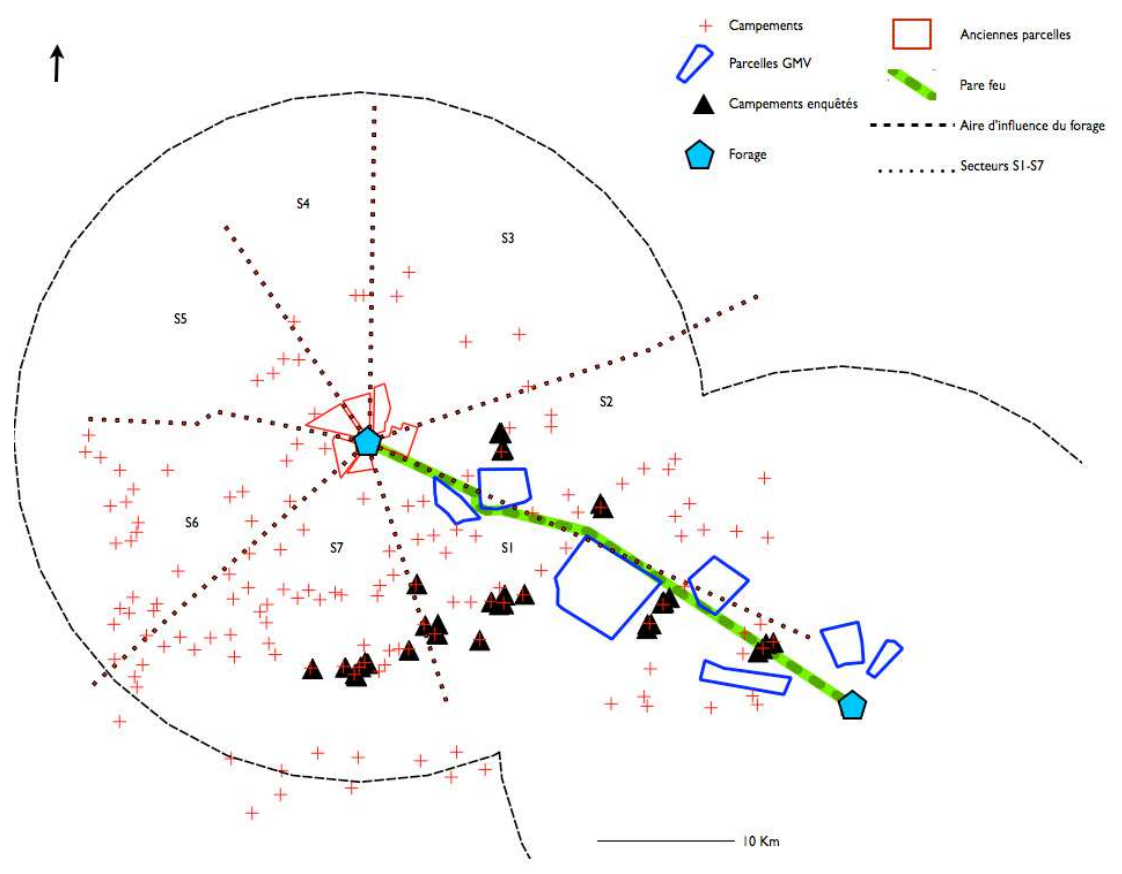

Figure 1 : Configuration géographique de la zone d'étude autour du forage de Widou Thiengoly.

\section{Communautés locales et institutions : relations interpersonnelles et création d'un ordre local}

La distance entre rhétorique participative et réalité locale, ajoutée au sentiment du peu de moyens disponibles et de leur répartition aléatoire, a progressivement fait émerger des interactions à huis clos entre acteurs influents, utilisant le cadre du projet dans une perspective redevenue personnelle. Cependant, la rhétorique reste utilisée par tous pour légitimer les choix scientifiques et les financements. Le défaut d'adhésion rend impossible les solutions rapides et radicales pour maintenir, adapter, voire réorganiser 
le processus d'action collective. Cette distanciation n'est pas propre au cadre de la GMV, mais constitue de fait un classique dans les projets de développement, en particulier en pays du Sud, de l'écart entre vœux et praticité (Biershenk et al., 2000 ; Bonnal et al., 1997, Blundo, 2001). Le vocable «participatif» utilisé souvent de manière incantatoire n’a de force au-delà des outils (Chambers, 1994; Loader \& Amartiya, 1999; Brown, 2006) que dans son acceptation complète : participation implique négociation, réinterprétation, multiplicité des acteurs, prise en compte des mécontents, des conflits et donc remise en cause et/ou affirmation des objectifs. Il s'agit d'abandonner du pouvoir (Saqalli, 2008) dans le sens où toute décision est soumise à la confrontation d'un nombre important d'acteurs. Le pouvoir d'agir ou non est conditionné par la manière dont sont négociées les relations entre des acteurs aux modèles mentaux hétérogènes (Turner, 1999; Mathevet et al., 2011).

Au sens d'Olivier de Sardan, le projet GMV est comme tout projet une « arène » où se gèrent les conflits, bien que, dans notre cas, ce terme occulte en partie un spectre de relations interindividuelles complexes menant à des situations non conflictuelles, via par exemple des processus de collusion entre éleveurs et agents de l'État pour l'attribution de parcelles. Nous observons une situation proche de ce que décrit la psychologie sociale par le terme "groupement d'individus» (Moscovici, 1984). Le qualificatif de groupement signifie que, bien que les membres du projet aient peu de contacts et de liens de routine opérationnels, la majorité des participants se reconnaissent via des réseaux politiques nationaux ou locaux (principalement via une compétition locale entre le Parti Démocratique Sénégalais de A. Wade et l'Union pour le renouveau démocratique de Djibo Ka, ministre de l'Environnement à l'époque de nos observations), claniques (Bissinaabe/Bacarnaabe), familiaux (népotisme pour le placement d'acteurs clés), scientifiques (thématiques ou institutionnels) ou techniques. Il y a permanence relative des objectifs dans l'intervalle des réunions programmées. Cependant, si l'objet de recherche et de développement est bien l'initiative GMV pour tous, il n'y a pas eu véritablement d'appropriation d'une identité collective « Grande Muraille Verte ». L'objectif commun n'est jamais clairement défini. La cohésion est en quelque sorte tacitement déléguée aux dirigeants et aux coordinateurs, favorisant implicitement une forme de hiérarchie alors même que la rhétorique institutionnelle incite à la participation des acteurs locaux. À l'intérieur de ce groupement organisé sous le chapeau " GMV », évoluent des membres régis par des systèmes décisionnels différents, qui cohabitent selon des représentations mentales hétérogènes. Les scientifiques utilisent un mode décisionnel anarchique, au sens politique du terme, aucun ne dominant l'autre en termes de décisions publiques. L'agence de la GMV utilise un style hiérarchique en accord avec le statut paramilitaire de ses membres, où les agents de terrain sont dominés par les décisions centrales du directeur basé à Dakar. Son pouvoir constitutif est basé sur le code de l'environnement, normatif et contraignant vis-à-vis des pratiques locales comme la coupe de bois par exemple. Ces décisions centrales sont néanmoins réappropriées par les acteurs locaux. La communauté rurale quant à elle est régie par 
un style proche de la coarchie (Lemieux, 1989) où les acteurs sont codominants dans les décisions publiques. Son pouvoir est indicatif, proche de l'influence. De ce schéma empirique de gouvernance résulte un système politique proche de la panarchie (De Puydt, 1860 ; Garmestani et al., 2009; Ruhl, 2012), où, de la coexistence de plusieurs systèmes politiques, résulte l'émergence d'un ordre local qui régit le cadre des décisions opérationnelles liées au projet.

En conséquence, l'évolution locale d'un tel groupement dépend presque entièrement des relations interpersonnelles entre acteurs, desquels découle la "réalité empirique » (comment se fait l'action collective au quotidien) ainsi que «l'image sociale du succès » (comment est légitimé le projet aux échelles bilatérale et internationale).

Dans ce contexte, les contraintes d'échelles décisionnelles (le passage des directives transnationales à l'action locale) sont moins un problème de communication qu'un blocage fondamental par des objectifs divergents. La capacité de négocier au quotidien la toile de l'action collective entre rhétorique institutionnelle et principe de réalité est laissée à l'interprétation des acteurs et constitue le véritable pouvoir d'action.

\section{Conclusion}

À partir de l'exemple d'un projet panafricain de lutte contre la désertification, nous avons interrogé la rhétorique institutionnelle au niveau international sur la protection intégrée de l'environnement en analysant sa mise en œuvre opérationnelle sur le terrain du Sahel. Par une approche réflexive d'observateurs participants, la place des chercheurs dans le système de gouvernance du projet de développement a été questionnée.

Les questions environnementales comme la désertification sont traitées au niveau politique à l'échelle de l'Union africaine. Cependant, au niveau de communautés, ces questions impliquent avant tout des changements d'organisations sociales appliquées à l'environnement. Dans ce contexte, le Millennium Ecosystem Assessment produit des recommandations de gestion dont l'injonction première est la participation des communautés locales à la protection de l'environnement, elle-même liée au développement socio-économique du Ferlo.

Nous avons montré que la participation des personnes concernées à la prise de décision sur des affaires communes comme l’implantation géographique des parcelles reboisées implique un mode de gestion locale par la coexistence des pouvoirs normatifs de l'État et des communautés locales. Alors que l'État administre le projet de reforestation via le code de l'environnement, les populations locales négocient des solutions individuelles qui maintiennent la paix sociale sur le terrain.

Finalement, le thème global de la désertification permet la mobilisation de fonds financiers internationaux et la rhétorique de la gouvernance, dont la définition est laissée volontairement à l'interprétation de chacun des acteurs, permet quant à elle les 
ajustements d'objectifs. La gouvernance est une manière de redéfinir un thème environnemental global en actions de gestion socio-écologiques contextualisées où chaque partie prenante trouvera un bénéfice à conserver le dialogue ouvert.

Le scientifique a quant à lui une place entière dans la gouvernance locale quand il est sollicité par les communautés sur le terrain et ceci, bien que les injonctions d'aide à la décision soient souvent hors de sa compétence technique et non suffisamment reconnue dans ses attributions professionnelles par les institutions scientifiques.

\section{Bibliographie}

Biershenk T., Chauveau J.-P., Olivier de Sardan J.-P. (2000), Courtiers en développement. Les villages africains en quête de projet, Paris, APAD-Karthala.

BINSWANGER H. (1986), «Evaluating research system performance and targeting research in land-abundant areas of sub-Saharian Africa », World Development, vol 14, p. 469-475.

Bonnal J., Chauveau J.-P., Lebas P., Lavigne-Delville P. (1997), La dynamique des sociétés rurales face aux projets participatifs de développement rural : réflexions et propositions d'action à partir d'expériences d'Afrique de l'ouest, Rome, FAO.

BROADBENT J. (2014), « Inter-disciplinary analysis of climate change and society : a network approach », in M. J. MANFReDo, J. J. VASKe, A. ReChKeMmeR, E. A. Duke (dir.) Understanding society and natural resources: forging new strands of integration across the social sciences, New York, Springer Open, p. 203-228.

BRown M. E. (2006), « Assessing natural resource management challenges in Senegal using data from Participatory Rural Appraisals and remote sensing », World Development, vol. 34, p. 751-767.

BLONDIAUX L. (2004), «L'idée de démocratie participative : enjeux, impensés et questions récurrentes », in M.-H. BACQUE, Y. SINTOMER (dir.), Démocratie participative et gestion de proximité, Paris, La Découverte, p. 119-137.

ChAmbers R. (1994), «Participatory rural appraisal (PRA): analysis of experience», World Development, vol. 22, p. 1253-1268.

DE Puydt P. E. (1860) «Panarchie», Revue Trimestrielle de Bruxelles, vol. 07-1860.

Garmestani A.S., Allen C.R., Gunderson L. (2009), «Panarchy: discontinuities reveal similarities in the dynamic system structure of ecological and social systems», Ecology er Society, vol. 14. En ligne, consulté le 30/12/2016. URL : http://dlc.dlib.indiana.edu/dlc/handle/10535/2642. 
Kristjanson P., Place F. M., Franzel S., Thornton P. K. (2002), « Assessing research impact on poverty: the importance of farmers' perspectives », Agricultural Systems vol. 72, p. 73-92.

LASDEL, Séries Études et Travaux. 1999-2016. En ligne, consulté le 30/12/2016. URL: http://www.lasdel.net/index.php/nos-activites/etudes-travaux.

LAVIGNE-Delville P. (1999), «Impasses cognitives et expertise en sciences sociales : réflexions à propos du développement rural en Afrique», Coopérer aujourd'hui, Les documents de travail de la Direction scientifique du GRET, vol. 9.

LAVigne-Delville P. (2000), «Regards sur les enquêtes et diagnostics participatifs : La situation d'enquête comme interface", Coopérer aujourd'hui, Les documents de travail de la Direction scientifique du GRET, $\mathrm{n}^{\circ} 17$.

LAVigne-Delville P. (2011), «Pour une anthropologie symétrique entre "développeurs" et “développés” », Cahiers D'études Africaines, vol. 202-203, n² 2-3, p. 491-509.

LEMIEUX V. (1989), La structuration du pouvoir dans les systèmes politiques, Québec, Les Presses de l’Université Laval.

LOADER R., AMARTIYA L. (1999), « Participatory Rural Appraisal: extending the research methods base », Agricultural Systems vol. 62, p. 73-85.

BLundo G. (2001), « Donnant Donnant. Les stratégies d'acteurs villageois face aux conditionnalités des projets de développement au Mali par M. $\square$ L. Mathieu, Thèse de doctorat en anthropologie de l'EHESS », Bulletin de l'APAD, vol. 22.

Mathevet R., Etienne M., Lynam T., Calvet C. (2011), Water management in the Camargue biosphere reserve: insights from comparative mental models analysis, Ecology \& Society, vol. 16. En ligne, consulté le 30/12/2016. URL : http://dlc.dlib.indiana.edu/dlc/handle/10535/7585.

Mosse D. (2005), Cultivating development: an ethnography of aid policy and practice (anthropology, culture and society Series), Londres, Pluto Press.

NEDERLOF S. E., DANGBÉGNON C. (2007), « Lessons for farmer-oriented research: Experiences from a West African soil fertility management project », Agriculture and $\mathrm{Hu}$ man Values, vol. 24, p. 369-387.

Olivier DE SARDAN J.-P., BOIRAL P., LANTERI J.-F. (1995), Paysans, experts et chercheurs en Afrique noire, sciences sociales et développement rural, Paris, CIFACE KARTHALA.

Olivier DE SARDAN J.-P., DAGOBI A. E. (2000), «La gestion communautaire sert-elle l'intérêt public ? Le cas de l'hydraulique villageoise au Niger », Politique africaine, vol. 80 , p. 153-68. 
OSTROM E. (1990), Governing the commons: the evolution of institutions for collective action, Cambridge, Cambridge University Press.

PARROTt L., MEYer W. S. (2012), «Future landscapes: managing within complexity », Frontiers in Ecology and the Environment, vol. 10, nº 7, p. 382-389.

Poussin J.-C., DiALlo Y., LegouPIL J.-C. (2006), « Improved collective decision-making in action for irrigated rice farmers in the Senegal River Valley ", Agricultural Systems $\mathrm{n}^{\circ} 89$, p. 299-323.

RuHL J.B. (2012), « Panarchy and the law», Ecology \& Society vol. 17. En ligne, consulté le 30/12/2016. URL : http://dlc.dlib.indiana.edu/dlc/handle/10535/8548.

SAQALLI M. (2008), «Le pouvoir des savoirs : enjeux et impacts des concepts sur le développement rural pour le Sahel nigérien», Vertigo, vol. 8, $\mathrm{n}^{\circ} 2$.

TURNER M. D. (1999), « No space for participation : pastoralist narratives and the ethiology of park-herder conflict in southeastern Niger ", Land Degradation e Development, vol. 10, $\mathrm{n}^{\circ} 4$, p. 345-363.

Weber M. (1971 [1921]), Économie et Société, Paris, Plon. 